\title{
AN ALGEBRAIC PERIODICITY THEOREM FOR SPHERES
}

\author{
JOSEPH P. BRENNAN ${ }^{1}$
}

\begin{abstract}
A periodicity theorem is given for spheres over a field of finite level generalizing results of Jouanolou. An extension of this result gives families of smooth affine varities with isomorphic $K$-groups.
\end{abstract}

The Bott periodicity theorem [1] lies at the foundation of topological $K$-theory. In particular, it yields the fact that the real and complex topological $K$-groups of spheres are periodic in the dimension. Consequent to work of Fossum [2] and Jouanolou [4], it was noted that the algebraic $K_{0}$-groups of complex spheres were isomorphic to the topological $K$-groups hence they enjoyed periodic behavior. Later Jouanolou [5] showed that if -1 is a square in the field $k$ and if $X_{n}=$ $\operatorname{Spec}\left(k\left[x_{0}, \ldots, x_{n}\right] /\left(1-\sum_{j=0}^{n} x_{j}^{2}\right)\right)$ then $K_{i}\left(X_{n}\right) \simeq K_{i}(k) \oplus K_{i}(k)$ if $n$ is even while $K_{i}(k) \oplus K_{i-1}(k)$ if $n$ is odd where $i$ is arbitrary and $K_{i}(k)=K_{i}(\operatorname{Spec} k)$.

In this paper we prove the following "algebraic periodicity" theorem which is an extension of Jouanolou's result. If $k$ is a field of characteristic not equal to two, $l(k)$, the level of the field $k$, is finite and $X_{n}$ as above, then $K_{i}\left(X_{n}\right) \simeq K_{i}\left(X_{n+2 l(k)}\right)$. This is Jouanolou's result when -1 is a square in $k$.

The author would like to thank the Matematisk Institut of Aarhus Universitet and the Institute for Algebraic Meditation for their hospitality during the preparation of this paper. The author would also like to thank R. Fossum and C. Weibel for their suggestions and corrections.

Consider the Witt ring $W(k)$ of a field $k$ [7]. The characteristic of this ring can be characterized by the concept of the level of a field. The level of a field $k, l(k)$, is the smallest integer $n$ such that -1 is the sum of $n$ squares.

Proposition [7]. The characteristic of $W(k)$ is $2 l(k)$ provided $l(k)<\infty$ and if $l(k)=\infty$ then the characteristic of $W(k)=0$.

THEOREM [8]. The level of a field is a power of two or infinite.

For a full discussion of the definitions and notations of $K$-theory we refer the reader to the papers of Quillen [9, 10] and Grayson [3]. We will, however, use the $G_{*}$-notation for the $K_{*}^{\prime}$-notation of [10], and remark that the following Lemma does not seem to appear in the literature.

Received by the editors January 3, 1983 and, in revised form, May 16, 1983.

1980 Mathematics Subject Classification. Primary 18F25; Secondary 10C05, 13D15, 14F15.

'This research was supported in part by the National Science Foundation and the University of Illinois Research Board. 
Lemma. Let $X$ be a separated Noetherian $k$-scheme with a $k$-rational point; then $K_{i}(k)$ is a direct summand of $K_{i}(X)$. If, moreover, $X$ is smooth projective, then $G_{i}(k) \simeq K_{i}(k)$ is a direct summand of $G_{i}(X)$.

Proof. Let $\tau: X \rightarrow$ Spec $k$ be the structure map and $\tau:$ Spec $k \rightarrow X$ the imbedding of the rational point. Then the composition $\tau \circ \lambda$ is the identity on Spec $k$. Hence the induced maps under $K_{*}$ give a splitting. If $X$ is smooth then $K_{i}(X) \simeq G_{i}(X)$, while if $X$ is projective then $\lambda, \tau$ and $\tau \circ \lambda$ are proper; hence, using [10, §7, 2.7] the induced maps under $G_{*}$ give a splitting.

For $X$ a scheme with $k$-rational point $\mathbf{x}$ and imbedding $\lambda$, denote the kernel of the $\operatorname{map} \lambda^{*}: K_{*}(X) \rightarrow K_{*}(\operatorname{Spec} k)$ by $T_{\mathbf{x}} K_{*}(X)$ (resp. denote the kernel of the map $\lambda^{*}$ : $G_{*}(X) \rightarrow G_{*}(\operatorname{Spec} k)$ by $\left.T_{\mathbf{x}} G_{*}(X)\right)$. Note that when $X$ is a $k$-scheme the isomorphism class of $T_{\mathbf{x}} K_{i}(X)$ is independent of $\mathbf{x}$ and we denote it by $T K_{i}(X)$ so that $K_{i}(X) \simeq K_{i}(k) \oplus T K_{i}(X)$. If further $X$ is smooth or projective then the isomorphism class of $T_{\mathbf{x}} G_{i}(X)$ is independent of $x$ and we denote it by $T G_{i}(X)$ so that $G_{i}(X) \simeq G_{i}(k) \oplus T G_{i}(X)$.

We are now in a position to address ourselves to the principal Theorem.

THEOREM. Suppose that $k$ is a field of characteristic not equal to two, that $l(k)<\infty$, and let $X_{n}=\operatorname{Spec}\left(k\left[x_{0}, \ldots, x_{n}\right] /\left(1-\sum_{i=0}^{n} x_{i}^{2}\right)\right)$. Then $K_{i}\left(X_{n}\right) \simeq K_{i}\left(X_{n+2 /(k)}\right)$.

Proof. For all $n, X_{n}$ is regular, hence $K_{i}\left(X_{n}\right) \simeq G_{i}\left(X_{n}\right)$. As char $W(k)=2 l(k)$ we have by the results on quadratic forms that

$X_{n+2 /(k)} \simeq \operatorname{Spec}\left(k\left[x_{0}, \ldots, x_{n}, u_{1}, \ldots, u_{l(k)}, v_{1}, \ldots, v_{l(k)}\right] /\left(1-\sum_{i=0}^{n} x_{1}^{2}-\sum_{j=0}^{l(k)} u_{j} v_{j}\right)\right)$.

For the sake of convenience let us denote

$$
Z_{\lambda}=\operatorname{Spec}\left(k\left[x_{0}, \ldots, x_{n}, u_{1}, \ldots, u_{l(k)}, v_{1}, \ldots, v_{\lambda}\right] /\left(1-\sum_{i=0}^{n} x_{1}^{2}-\sum_{j=1}^{\lambda} u_{j} v_{j}\right)\right) \lambda \geqslant 1
$$

and let

$$
Z_{0}=\operatorname{Spec}\left(k\left[x_{0}, \ldots, x_{n}, u_{1}, \ldots, u_{l(k)}\right] /\left(1-\sum_{i=0}^{n} x_{1}^{2}\right)\right) \simeq X_{n}\left[u_{1}, \ldots, u_{l(k)}\right],
$$

and let

$$
U_{\lambda}=\operatorname{Spec}\left(k\left[x_{0}, \ldots, x_{n}, u, \ldots, u_{\lambda}, \ldots, u_{l(k)}, v_{1}, \ldots, v_{\lambda}, v_{\lambda}^{-1}\right]\right) \text { for } \lambda \geqslant 1 .
$$

We note that $Z_{\lambda}$ and $U_{\lambda}$ are regular. Then by Quillen's localization theorem (see [3, p. 299; 9, Theorem 4; 10, §7, Proposition 3.2]), we have for $f: Z_{\lambda} \rightarrow Z_{\lambda+1}$ and $j$ : $U_{\lambda+1} \rightarrow Z_{\lambda+1}$ the natural immersions, the long exact sequence

$$
\cdots \stackrel{\partial}{\rightarrow} K_{i}\left(Z_{\lambda}\right) \stackrel{f_{*}}{\rightarrow} K_{i}\left(Z_{\lambda+1}\right) \stackrel{j^{*}}{\rightarrow} K_{i}\left(U_{\lambda+1}\right) \stackrel{\partial}{\rightarrow} K_{i-1}\left(Z_{\lambda}\right) \stackrel{f_{*}}{\rightarrow} K_{i-1}\left(Z_{\lambda+1}\right) \stackrel{j^{*}}{\rightarrow} .
$$

$Z_{\lambda}$ is a $k$-scheme with a $k$-rational point. Let $\tau: Z_{\lambda} \rightarrow$ Spec $k$ be the structure map and let $\pi$ : Spec $k \rightarrow Z_{\lambda}$ be the imbedding. $U_{\lambda}$ is also a $k$-scheme with a $k$-rational point; let $\gamma$ : Spec $k \rightarrow U_{\lambda}$ be the imbedding. Further, by the fundamental theorem of $K$-theory $[3,10], K_{i}\left(U_{\lambda}\right) \simeq K_{i}(k) \oplus K_{i-1}(k)$. 
The composition Spec $k \stackrel{\gamma}{\rightarrow} U_{\lambda+1} \stackrel{j}{\rightarrow} Z_{\lambda+1} \stackrel{\tau}{\rightarrow}$ Spec $k$ is the identity on Spec $k$, hence $\left.j^{*}\right|_{K_{1}(k)}$ is an isomorphism onto the complement of $K_{i-1}(k)\left(=T K_{i}\left(U_{\lambda+1}\right)\right)$. Moreover, the composition

$$
\operatorname{Spec} K \stackrel{\pi}{\rightarrow} Z_{\lambda} \stackrel{f}{\rightarrow} Z_{\lambda+1} \stackrel{\tau}{\rightarrow} \operatorname{Spec} k
$$

is the identity on Spec $k$, hence $\left.f^{*}\right|_{K_{i}(k)}$ is an isomorphism onto the complement of $T K_{i}\left(Z_{\lambda}\right)$. Kato's theorem on boundary map [6, \$2.4] says that $\left.\partial\right|_{K_{i}(k)}=\left.f^{*}\right|_{K_{i}(k)}$, hence $\partial$ maps onto the complement of $T K_{i}\left(Z_{\lambda}\right)$. Therefore we have that $T K_{i}\left(Z_{\lambda}\right) \simeq$ $T K_{i}\left(Z_{\lambda+1}\right)$ by restriction of the map $f_{*}$. So $K_{i}\left(Z_{\lambda}\right) \simeq K_{i}\left(Z_{\lambda+1}\right)$ for all $\lambda$ and $i$. But $K_{i}\left(Z_{0}\right) \simeq K_{i}\left(X_{n}\right)$ by the fundamental theorem [3,10] while $Z_{l(k)} \simeq X_{n+2 l(k)}$. So $K_{i}\left(X_{n+2 l(k)}\right) \simeq K_{i}\left(X_{n}\right)$.

An examination of the above proof indicates that we have proved more

THEOREM. Let $Y=\operatorname{Spec}\left(k\left[x_{0}, \ldots, x_{n}\right] / p(\mathbf{x})\right)$ be regular and let

$$
V=\operatorname{Spec}\left(k\left[x_{0}, \ldots, x_{n} u, v\right] / p(\mathbf{x})+u v\right) .
$$

Then $K_{i}(Y) \simeq K_{i}(V)$.

We immediately obtain

Corollary. (1) If $l(k) \neq \infty$ and $n \equiv 0 \bmod 2 l(k)$ then $K_{i}\left(X_{n}\right) \simeq K_{i}(k) \oplus K_{i}(k)$

(2) If $l(k) \neq \infty$ and $n \equiv-1 \bmod 2 l(k)$ then $K_{i}\left(X_{n}\right) \simeq K_{i}(k) \oplus K_{i-1}(k)$.

Proof. (1) By induction it is enough to show this is true for $n=0$. But $X_{0}=\operatorname{Spec}\left(k\left[x_{0}\right] /\left(1-x_{0}^{2}\right)\right)$ and $k\left[x_{0}\right] / 1-x_{0}^{2} \simeq k \oplus k$, hence $K_{i}\left(X_{0}\right) \simeq K_{i}(k) \oplus$ $K_{i}(k)$.

(2) Again by induction it is enough to show this is true for $n=2 l(k)-1$. But $X_{2 l(k)-1} \simeq \operatorname{Spec}\left(k\left[u_{1}, \ldots, u_{l(k)}, v_{1}, \ldots, v_{l(k)}\right] /\left(1-\sum_{j=1}^{l(k)} u_{j} v_{j}\right)\right)$, hence

$$
\begin{aligned}
K_{1}\left(X_{2 l(k)-1}\right) & \simeq K_{i}(\operatorname{Spec} k[u, v] /(1-u v)) \\
& \simeq K_{i}\left(\operatorname{Spec} k\left[u, u^{-1}\right]\right) \simeq K_{i}(k) \oplus K_{i-1}(k) .
\end{aligned}
$$

This Corollary is the result proved by Jouanolou [5].

During the preparation of this paper, the author has become aware of recent work by A. Suslin [11, Theorem 2.3] proving part (2) of the Corollary by similar methods.

\section{REFERENCES}

1. M. Atyah, K-theory, Benjamin, New York and Amsterdam, 1967.

2. R. Fossum, Vector bundles over spheres are algebraic, Invent. Math. 8 (1969), 222-225.

3. D. Grayson, Higher algebraic K-theory. II, Algebraic K-Theory (M. R. Stein, ed.), Evanston, 1976, Lecture Notes in Math., vol. 551, Springer-Verlag, Berlin, Heidelberg and New York, 1976, pp. 217-240.

4. J.-P. Jouanolou, Comparison des K-théories algébrique et topologique de quelques variétés algébrique, C. R. Acad. Sci. Paris Sér A 272 (1971), 1373-1375.

5. __ Quelques calculs en $K$-theorie des schemes, Algebraic K-Theory. I (H. Bass, ed.), Lecture Notes in Math. vol. 341, Springer-Verlag, Berlin, Heidelberg and New York, 1973, pp. 317-335.

6. K. Kato, A generalization of local class field theory using $K$-groups. II, J. Fac. Sci. Univ. Tokyo Sect. IA Math 27 (1980), 603-683.

7. T. Lam, The algebraic theory of quadratic forms, Benjamin, Reading, Mass., 1973.

8. A. Pfister, Zur Darstellung von - 1 Als Summe von Quadraten in einem Körper, J. London Math. Soc. Ser. I 40 (1965), 159-165. 
9. D. Quillen, Higher K-theories for categories with exact sequences, New Developments in Topology (G. Segal, ed.), London Math. Soc. Lecture Notes Ser., No. 11, Cambridge Univ. Press, London and New York, 1974, pp. 95-103.

10. __ Higher algebraic K-theory. I, Algebraic K-Theory. I (H. Bass, ed.), Lecture Notes in Math., vol. 341, Springer-Verlag, Berlin, Heidelberg and New York, 1973, pp. 85-147.

11. A. A. Suslin, Mennicke symbols and their applications in the $K$-theory of fields, Algebraic $K$-Theory (R. Keith Dennis, ed.), Lecture Notes in Math., vol. 966, Springer-Verlag, Berlin, Heidelberg and New York, 1982, pp. 331-356.

Department of Mathematics, University of Illinois, Urbana, Illinois 61801

Current address: Department of Mathematics, Michigan State University, East Lansing, Michigan 48824 